\title{
Elaboración de ladrillos ecológicos en muros no estructurales: una revisión
}

\section{Making ecological bricks in non-structural walls: a review}

Sócrates Pedro Muñoz-Pérez¹, José Luis Delgado-Sánchez¹, Luis Enrique Facundo-Peña ${ }^{1}$

1 Universidad Señor de Sipán

\section{RESUMEN}

El ladrillo es uno de los materiales más usados en el mundo, cuyo proceso de fabricación consume recursos naturales, como los combustibles, que generan calor y contaminación. Los muros no portantes son aquellos que no soportan cargas debido a que actúan como divisiones en una edificación, en tanto que los muros de mampostería se utilizan como tabiquería cuando la edificación es alta, debido a que, conforme el muro esté en un piso más alto, las cargas que llegan a recibir estos se reducen en un $40 \mathrm{y}$ un $60 \%$. Este documento tiene por objetivo demostrar la investigación relevante publicada en revistas indexadas de los años 2015 a 2020, de los cuales 33 artículos fueron de Scopus, 13 de Scielo, 2 de ScienceDirect y 2 de Redalyc, sobre la elaboración del ecoladrillo en muros no estructurales con diferentes desechos sólidos, presentando también los resultados de los trabajos realizados en el ámbito, para poder de esta manera ver el impacto que cada uno produce en la construcción y el medio ambiente.

PALABRAS CLAVE: construcción; desechos sólidos; ecoladrillos; medio ambiente; muros no portantes.

\section{ABSTRACT}

Brick is one of the most widely used materials in the world, whose manufacturing process consumes natural resources, such as fuels, which generate heat and pollution. Non-bearing walls are those that do not support loads because they act as divisions in a building, while masonry walls are used as partitions when the building is high, because, as the wall is on a higher floor, the loads that they receive are reduced by 40 and $60 \%$. The objective of this document is to demonstrate the relevant research published in indexed journals from 2015 to 2020, of which 33 articles were from Scopus, 13 from Scielo, 2 from ScienceDirect and 2 from Redalyc, on the development of eco-brick in non-structural walls with different solid waste, also presenting the results of the work carried out in the area, in order to see the impact that each one produces on construction and the environment..

KEYWORDS: construction; solid waste; eco-bricks; environment; non-bearing walls.

\section{Correspondencia:}

DESTINATARIO: Sócrates Pedro Muñoz Pérez INSTITUCIÓN: Universidad Señor de Sipán DIRECCIÓN: Km 5 Carretera a Pimentel, C. P. 1400, Chiclayo - Perú CORREO ELECTRÓNICO: msocrates@crece.uss.edu.pe
Fecha de recepción: 12 de noviembre de 2020. Fecha de aceptación: 5 de febrero de 2021. Fecha de publicación: 10 de febrero de 2021.
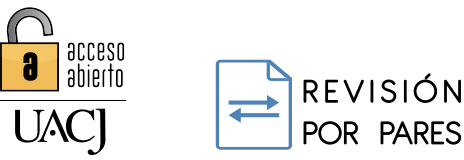

Licencia Creative Commons

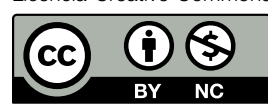




\section{INTRODUCCIÓN}

Uno de los problemas sociales en los países en desarrollo es la falta de vivienda y materiales de construcción a precios asequibles, entre los cuales destaca el ladrillo, que es uno de los materiales de mayor importancia y contaminación [1]. En la actualidad, la fabricación de ladrillos huecos de arcilla cocida no solo tienen un impacto negativo para el ambiente, sino que también se caracterizan por la conductividad térmica y la absorción de agua ${ }^{[2]}$.

En 2010 se generaron 1300 toneladas de residuos sólidos en el mundo, esperando que para 2025 aumente alrededor de 2200 millones de toneladas, y los desechos inorgánicos, que incluyen plástico, papel, vidrio y otros materiales, representan el $72 \%$ del total desechos sólidos en los países de altos ingresos y el 36\%-46\% en los de medios y bajos ingresos ${ }^{[3]}$.

En Colombia, en 2014 se generaron alrededor $13.8 \mathrm{mi}-$ llones de toneladas de residuos sólidos, de los cuales el 61.5\% fue de residuos sólidos orgánicos [4]. En la India empeoran los problemas ambientales, ya que en 2015 se generaron 0.143 millones de desechos sólidos por día, siendo el plástico el más resaltante [5].

En China, la cerámica tiene como tratamiento los residuos sólidos de las tierras, por lo cual estos desechos ocasionan un gran problema ambiental [6]. En Italia, las fábricas que se dedican a producir cerámica son de las que más contaminan y uno de los residuos que más resalta es el lodo de cerámica, que el país ha decido añadirlo como aditivo en la elaboración de ladrillos [7].

La ciudad de Tirupur es contaminada por agua, tierra y aire, debido a las 830 unidades textiles con ocho plantas de tratamiento, las cuales producen un lodo que contamina al suelo y agua con estas sustancias químicas, surgiendo la idea de crear un ladrillo adhiriendo el lodo [8]. En la ciudad de Bogotá, los residuos con plomo (chatarra) están provocando gran contaminación debido a que causan daño no solo en el aire, sino también en la tierra y el agua, de tal manera que se estudió estas escorias metalúrgicas para que sean utilizadas como aditivo en la elaboración de ladrillos [9].

En México se generan aproximadamente 33666 toneladas diarias de desechos de la construcción, de las cuales solo se reciclan 1000 toneladas diarias y lo que resta termina provocando problemas ambientales en la población ${ }^{[10]}$.

La generación de residuos sólidos está aumentando en muchos sectores industriales, por cual se requieren medidas efectivas para garantizar un descarte que sea ambientalmente adecuado y económicamente viable [11].

\section{METODOLOGÍA}

Para la presente revisión de literatura se investigó en bases de datos y ahí se encontraron los siguientes artículos indexados: 33 en Scopus, 13 en Scielo, 2 en ScienceDirect y 2 en Redalyc, de los cuales 17 son de 2020, 11 de 2019, 3 de 2018, 9 de 2017, 7 de 2016 y 3 de 2015. Para la búsqueda se utilizaron palabras claves como construcción, desechos sólidos, ecoladrillos, medio ambiente, muros no portantes, eco-brick, bricks, ecological bricks, ladrillos sostenibles, ladrillos, sustainable bricks, interlocking bricks y tijolos. Para un mejor detalle, en la Tabla 1 se muestran los artículos distribuidos, según base de datos y año de publicación.

TABLA 1

Distribución de los Artículos Usados Como Referencia, Según Base de Datos y año de Publicación

\begin{tabular}{|c|c|c|c|c|c|c|c|}
\hline \multirow{2}{*}{ BASE DE DATOS } & \multicolumn{6}{|c|}{ AÑo DE PUBLICACIÓN } & \multirow{2}{*}{ TOTAL } \\
\hline & 2015 & 2016 & 2017 & 2018 & 2019 & 2020 & \\
\hline Scopus & & 5 & 5 & 3 & 4 & 16 & 33 \\
\hline Scielo & 3 & 1 & 2 & & 7 & & 13 \\
\hline ScienceDirect & & 1 & & & & 1 & 2 \\
\hline Redalyc & & & 2 & & & & 2 \\
\hline Total & 3 & 7 & 9 & 3 & 11 & 17 & 50 \\
\hline
\end{tabular}

\section{RESULTADOS Y DISCUSIÓN}

\section{A. Daño al medio ambiente}

El ladrillo es uno de los materiales de construcción que se han empleado a lo largo de la historia. Su uso facilita el cerramiento, la protección y el aislamiento térmico y acústico de toda construcción [12].

En Bangladesh se fabrican alrededor de 17000 millones de ladrillos en hornos que consumen cerca de 4000 millones de toneladas de leña y carbón principalmente. Estas emisiones contribuyen en forma significativa al calentamiento global ${ }^{[13]}$.

El proceso de fabricación de ladrillos convencional aporta su parte consumiendo energía en la cocción, lo 
que acelera el calentamiento global y las enfermedades cardio-respiratorios atribuidas a la concentración de gases de efecto invernadero [14].

Se considera que el sector construcción es el que más daño ambiental ha causado en la Tierra y su hábitat, que además propicia un gran consumo de materia prima y energía [15]. En su proceso de fabricación, se consume un promedio de $2 \mathrm{kWh}$ (kilovatios-hora) por ladrillo y se genera cerca de $0.41 \mathrm{~kg}$ de $\mathrm{CO}_{2}$ (dióxido de carbono), produciendo grandes impactos ambientales ${ }^{[16]}$.

En Colombia, los residuos sólidos son los que más se producen y también los que menos se aprovechan [17].

En el proceso de cocción, el ladrillo requiere como combustible varios recursos naturales que están escaseando [18]. Actualmente, un tercio de la Tierra está compuesta de bosques (aproximadamente 2.98 millones de $\mathrm{km}^{2}$ ), de lo cual 23 millones de toneladas métricas de residuos de madera causan un gran daño al ambiente [19]. Los aserraderos producen grandes cantidades de aserrín y este provoca daño al entrar en contacto con los ojos de las personas ${ }^{[20]}$.

Algunas de las razones por las que hay malas prácticas de eliminación de residuos provocados por la construcción y demolición son el largo lapso entre el tiempo en que el usuario deja los residuos en la zona indicada y el momento en que son retirados por la empresa recolectora; el tipo incorrecto de residuo que el usuario deja en el punto de recolección que luego no recoge la empresa contratada; una legislación deficiente y, lo que es más importante, una aplicación deficiente de la ley por parte de las autoridades [21].

\section{B. Material reciclado utilizado en la industria de la construcción}

La alta demanda de recursos naturales debido a la rápida urbanización y el problema de eliminación de desechos en los países desarrollados, ha creado oportunidades para el uso de estos residuos en la industria de la construcción ${ }^{[22]}$.

En la Tabla 2 se muestra los porcentajes óptimos de remplazo de materiales reciclados que van a sustituir parcialmente a los componentes empleados en la fabricación de ladrillos. Como remplazo del agregado fino están los desechos de tallos de sorgo, que mejoran la resistencia a la compresión y aislación térmica; el polvo de mármol, que mejora la resistencia a compresión; el caucho, que mejora la absorción del agua; la cáscara de arroz y maní, que mejoran la resistencia a la compresión.

Como remplazo del cemento se tiene a los desechos de aceituna, que mejoran la aislación térmica; los de piedra y las cenizas de bagazo de caña de azúcar, que mejoran la resistencia a la compresión; el polvo de acero, que mejora la resistencia a la flexión y ayuda a la permeabilidad; la ceniza de estiércol de vaca y la ceniza de cáscara de arroz, que mejoran la resistencia a la compresión.

Por último, como remplazo de la arcilla está la paja, que mejora la resistencia a la compresión; el aserrín, que mejora la absorción del agua, la aislación térmica y la absorción sísmica; el café molido, que mejora la absorción del agua y aislación térmica, y el papel, que mejora la absorción del agua.

TABLA 2

Materiales de Construcción

\begin{tabular}{|c|c|c|}
\hline $\begin{array}{l}\text { MATERIAL } \\
\text { REMPLAZADO }\end{array}$ & MATERIAL RECICLADO & $\begin{array}{l}\text { PORCENTAJE } \\
\text { ÓPTIMO A } \\
\text { REMPLAZAR }\end{array}$ \\
\hline \multirow{5}{*}{$\begin{array}{l}\text { Agregado } \\
\text { fino }\end{array}$} & Desecho de tallos de sorgo & 5 \\
\hline & Polvo de mármol & 2 \\
\hline & Caucho & 20 \\
\hline & Cáscara de arroz & 4 \\
\hline & Cáscara de maní & 3 \\
\hline \multirow[t]{6}{*}{ Cemento } & Desechos de aceituna & 5 \\
\hline & Residuos de piedra & 4 \\
\hline & $\begin{array}{l}\text { Cenizas de bagazo de caña de } \\
\text { azúcar }\end{array}$ & 19 \\
\hline & Polvo de acero & 20 \\
\hline & Ceniza de estiércol de vaca & 5 \\
\hline & Ceniza de cáscara de arroz & 10 \\
\hline \multirow[t]{4}{*}{ Arcilla } & Paja & 3 \\
\hline & Aserrín & 3 \\
\hline & Café molido & 17 \\
\hline & Papel & 17 \\
\hline
\end{tabular}

\section{Material reciclado para la elaboración de ladrillos ecológicos}

El desarrollo sostenible de la economía mundial fuertemente industrializada ha llevado a un incremento sensible en la reutilización de residuos de producción. Este 
fenómeno se debe en gran parte a la fuerte especialización de los distintos sectores industriales [23].

Los desechos de conchas de ostras están siendo aprovechados para remplazar parcialmente al cemento y los desechos de sorgos (tallos de maicillo) para sustituir al agregado fino parcialmente y que de esta manera se pueda crear ecoladrillos y reducir la contaminación ambiental que estos desechos generan ${ }^{[24]}$.

El aserrín es un desecho de los aserraderos y la paja lo es de la caña de cereal, por lo cual están siendo utilizados como aditivo en la fabricación del ecoladrillo, remplazando en determinados porcentajes al agregado fino. $\mathrm{Al}$ añadir la paja se provoca aislación térmica y reducción de poros, y al agregar aserrín aumenta los poros y disminuye el aislamiento térmico. El aserrín agregado no debe exceder el 3\% [25], [26], [27].

Actualmente, las propiedades térmicas de las edificaciones es un asunto muy importante para las personas que habitan en ellas, debido a que el aire acondicionado y la calefacción consumen mucha energía. Por ello surgió la idea de fabricar ladrillo agregando café molido, ya que es el segundo material más comercializado en el mundo que produce una buena aislación térmica al utilizarlo como aditivo [28].

Las llantas, que son de los desechos sólidos que más preocupación han causado a los ambientalistas porque contienen materiales que tardan cientos años en deteriorarse, son convertidas en gránulos de goma para adicionarlos como agregado fino en la elaboración de los ecoladrillos, dando una mayor absorción y menor resistencia [29].

Con el fin de mejorar las propiedades térmicas, se realizó un estudio con fibras de palmera datilera, paja y desechos de aceituna, el cual dio buenos resultados y ayudó a reducir el consumo de calefacción y refrigeración ${ }^{[30]}$. Y otro estudio se llevó a cabo con residuos de dátiles, obteniendo un ahorro energético del $68 \%$ en el aire acondicionado [31].

También se probó la viabilidad del uso de residuos de piedra de dimensión (caliza), mezclándola con resina de poliéster para producir así un ladrillo ecológico que aumentó la resistencia al fuego [32].
Con el fin de mitigar el daño ambiental y reducir la explotación de bancos de arcilla provocada por la producción de ladrillo cocido, se fabricó ladrillo sustituyendo parcialmente la arcilla por cenizas de bagazo de caña de azúcar, con lo cual redujo el uso de arcilla hasta en un $30 \%[33]$.

En otro estudio ${ }^{[34]}$ se fabricó ladrillo ecológico con arcilla y contaminantes de polvo de acero, ya que al adicionar este se redujo la temperatura de cocción. Este producto cumple con las normativas comerciales estándares.

La ceniza del estiércol de vaca se usa para fabricar ladrillos ecológicos baratos, ayudando al planeta y en la parte económica ${ }^{[35] .}$

Por otra parte, se fabricó ladrillo con las cenizas de la cáscara de arroz, con buenos resultados y la reducción del costo de producción entre un 14\% al 42.8\% [36].

La utilidad de los ladrillos se ha diversificado y ahora los hay permeables y ambientalmente amigables. En algunos se usa la escoria de acero en su elaboración, lo cual es beneficioso para el ambiente, son ladrillos no quemados y no causa contaminación secundaria [37].

El papel es uno de los mayores residuos que se producen en el mundo, lo cual ha causado un gran daño a la humanidad y al ambiente. Por ello, se decidió aprovecharlo en la fabricación del ladrillo e incorporarlo en remplazo de la arcilla, obteniéndose una mayor absorción de agua debido al aumento de poros, una menor conductividad térmica y una menor resistencia a la compresión [38].

\section{Accesibilidad a los ladrillos ecológicos}

Actualmente, la tecnología en la construcción ha avanzado bastante y ha reducido el tiempo de las obras. Sin embargo, el gran problema para una parte de la población que vive en zonas rurales es el precio de los materiales, especialmente el ladrillo. Aparte de que no es tan asequible por su precio, es un material que en las obras de construcción deja demasiados desechos que hacen mucho daño a las personas y al ambiente. Por ello surgió la idea de crear un ladrillo con las materias primas que se encuentren disponibles localmente, como es la tierra, que es el material principal, una pequeña porción 
de cemento y desechos de la edificación, dando como resultado un material resistente, ecológico, seguro y barato [39], [40], [41].

El costo del ladrillo de arcilla es más elevado que el del ecoladrillo. Los recursos no renovables, como la arcilla, representan $33.5 \%$ del insumo de la producción del ladrillo, la energía el $18.1 \%$ y el servicio laboral (mano de obra y servicio) el $25.9 \%$, lo cual impacta en precios mayores a los del ladrillo ecológico que no necesita del proceso de cocción ni tampoco de otros recursos naturales, sino que solo se utiliza el suelo, el cemento y un material reciclado, genera menor contaminación ambiental y consume menos energía y servicio laboral. De esta manera, el costo del ladrillo ecológico se reduce en un $41 \%$ respecto al ladrillo de arcilla ${ }^{[42]}$.

En la actualidad hay una gran iniciativa de construcciones sostenibles, lo cual ha motivado a investigadores a sustituir materiales comúnmente usados en el concreto, cambiándolos parcialmente por materiales que por lo general son desechados, tales como residuos industriales, llantas, tuberías, vidrios, bolsas plásticas. Con ellos se hacen diferentes mezclas para mortero y para la posterior fabricación de ladrillo, con el fin de abaratar sus costos y disminuir los desechos [43].

Se ha realizado varios estudios para la reducir los costos a la hora de construir, como el ecobloque, que está elaborado con botellas de tereftalato de polietileno (PET, por sus siglas en inglés), y también se realizó una investigación con el cartón corrugado para usarlo como aislante térmico en viviendas, siendo un material de bajo costo [44], [45].

\section{E. Muros no estructurales}

Los muros no estructurales son aquellos que están requeridos por cargas verticales (peso propio) y horizontales (sismo) perpendiculares a su plano [46].

Los muros no estructurales pueden ser desplazados de un lugar a otro por variaciones en el uso de los espacios, originando, aún durante sismos moderados, una interacción pórtico-relleno que puede alterar significativamente la concepción de los diseños convencionales [47].

En el terremoto ocurrido en la Ciudad de México el 19 de septiembre de 2017 se observaron los daños que se produjeron en las fachadas y muros divisorios de las edificaciones, que se les considera como no estructurales, para así hacer cambios en las normativas de un comportamiento aceptable no solo para los sismos de diseño, sino para menor intensidad. Por tanto, ese estudio llevó a concluir que es conveniente tener una estructura rígida para limitar desplazamientos laterales durante los terremotos y reducir daños, y así evitar que se produzca el efecto de planta baja débil (muros que se interrumpen en la planta baja) [48].

Asimismo, en febrero del 2017 en la ciudad de Medellín, Colombia, un muro de mampostería de ladrillo no reforzada, colapsó bajo fuerzas de vientos moderadas por lo que sirvió para realizar un análisis acerca de estos modelos [49].

Los muros de mampostería se utilizan como tabiquería cuando la edificación es alta, debido a que la capacidad de estos muros disminuye con la altura. Los muros de mampostería no portantes pueden llegar a agrietarse, dándose hendiduras importante que reducen la capacidad de carga entre un 40 y un $60 \%$. Estas grietas se dan debido a la tensión de tracción de los elementos de concreto que los rodean, ya que están sujetos a deformación, por lo que los muros de mampostería muy rígidos pueden aplastar la estructura de concreto [50].

\section{CONCLUSIONES}

El ladrillo ecológico produce un impacto ambiental mayormente positivo, debido que está hecho de una mezcla conformada por suelo, agua y cemento, más un material reciclado como aditivo remplazante en cualquiera de sus componentes, para luego ser prensado a temperatura ambiente, el cual adquiere diferentes características que depende del material reciclado a remplazar parcialmente. En cambio, el ladrillo tradicional produce un impacto ambiental negativo debido a su proceso de cocción que emite a la atmósfera aproximadamente $0.41 \mathrm{~kg}$ de $\mathrm{CO}_{2}$ por ladrillo producido.

El ladrillo ecológico es una alternativa de solución para las personas de bajos recursos económicos en zonas alto andinas, ya que usando estos materiales, adicionados con material de desechos de tallos de sorgo, aserrín y café molido, mejoraría la aislación térmica de las viviendas.

El ladrillo ecológico es una alternativa de solución para personas que habitan en zonas rurales, calurosas y llu- 
viosas que al usar ladrillos ecológicos adicionados con papel, caucho, aserrín y café molido, mejorarían la absorción del agua y aislación térmica.

El ladrillo ecológico también es una alternativa de solución para la construcción de viviendas en zonas sísmicas, ya que se puede usar ladrillos ecológicos adicionados con aserrín para mejorar la aislación sísmica.

\section{REFERENCIAS}

[1] C. Arredondo-Orozco, M. Luna-del Risco, S. Villegas-Moncada, M. Gónzales-Palacio, C. Arrieta-Gónzales, J. Cutindioy-Imbachi, J. Aguirre-Morales y F. Quintero-Suárez, "A novel energy-efficient machine to compress inorganic residues in eco-bricks as a sustainable construction strategy for low-cost housing", 2019 14th Iberian Conference on Information Systems and Technologies (CISTI), Coimbra, Portugal, 2019, pp. 1-6, DOI: 10.23919/CISTI.2019.8760797.

[2] M. Šveda, B. Janík, V. Pavlík, Z. Štefunková, G. Pavlendová, P. Šín, R. Sokolář, "Pore-size distribution effects on the thermal conductivity of the fired clay body from lightweight bricks", J. Build. Phys., vol. 41, no. 1, pp. 7894, 1 jul. 2017. DOI: 10.1177/1744259116672437.

[3] F. C. Antico, M. J. Wiener, G. Araya-Letelier, R. Gonzalez-Retamal, "Eco-bricks: A sustainable substitute for construction materials", Rev. Ing. de Construcción, vol. 16, no. 3, pp. 518-526, 2017. DOI: 10.7764/ RDLC.16.3.518.

[4] R. Sánchez-Bernal, D. J. Pita-Castañeda, K. D. González-Velandia y J. A. Hormaza-Verdugo, "Análisis de mezclas de residuos sólidos orgánicos empleadas en la fabricación de ladrillos ecológicos no estructurales", $R C A$, vol. 53 , no. 1 , pp. 23-44, enero-junio 2019. DOI: 10.15359/rca.53-1.2.

[5] H. T. Mohan, L. Masson, S. Kolathayar, A. K. Sharma, Monish , A. G. Krishnan, S. Thiviya y R. Mohan, "Transforming urban waste into construction blocks for a sanitation infrastructure: A step towards addressing rural open defecation", 2017 IEEE Global Humanitarian Technology Conference (GHTC), San Jose, CA, 2017, pp. 1-9, DOI: 10.1109/GHTC.2017.8239291.

[6] Z. Yang, Z. Qiang, M. Guo, G. Yi, Y. Shi, F. Cheng y M. Zhang, "Pilot and industrial scale tests of high-per- formance permeable bricks producing from ceramic waste", J. Clean. Prod., vol. 254, pp. 1-9, 1 may. 2020. DOI: 10.1016/j.jclepro.2020.120167.

[7] C. Coletti, L. Maritan, G. Cultrone y C. Mazzoli, "Use of industrial ceramic sludge in brick production: Effect on aesthetic quality and physical properties", Constr Build Mater., vol. 124, pp. 219-227, 15 oct. 2016. DOI: 10.1016/j.conbuildmat.2016.07.096.

[8] V. T. Ashok-Kumar, P. T. Ravichandran y P. R. Kannan-Rajkumar, "Use of Textile Effluent Treatment Plant Sludge as Sustainable Material in Brick Manufacturing". En: M. Bhaskar, S. Dash, S. Das y B. Panigrahi (eds.), International Conference on Intelligent Computing and Applications. Advances in Intelligent Systems and Computing, vol 846, Springer, Singapore, pp. 283-291, 2019. DOI: 10.1007/978-981-13-2182-5_27.

[9] J. Santacruz-Torres y J. Torres-Agredo, "Aprovechamiento de escorias de fundición secundaria de plomo en ladrillos cerámicos", Cien. Ing. Neogranadina, vol. 29, no. 1, pp. 7-18, ag. 2019. DOI: 10.18359/rcin.3495.

[10] M. N. Rojas-Valencia y E. Aquino, "Recycling of construction wastes for manufacturing sustainable bricks", Proceedings of the Institution of Civil Engineers - Construction Materials, vol. 172, no. pp. 29-36, feb. 2019. DOI: $10.1680 /$ jcoma.16.00046.

[11] L. Simão, D. Hotza, F. Raupp-Pereira, J. Labrincha y O. Montedo, "Characterization of pulp and paper mill waste for the production of waste-based cement", Rev. Int. de Contam. Ambient., vol. 35, no. 1, 2019. DOI: 10.20937/RICA.2019.35.01.17.

[12] G. C. Restrepo-Zapata y C. F. Cadavid-Restrepo, "Improvement of Environmental and Energetic Performance of Social Housing in Medellin by Using Modified Ceramic Bricks", Rev. Ing. Univ. Medellín, vol. 18, no. 35, 2019. DOI: 10.22395/rium.v18n35a3.

[13] S. Kumar, M. Zhao, H. Zhang, M. A. Rahman, C. Luo "Distribution, contamination status and source of trace elements in the soil around brick kilns", Chemosphere, vol. 263, pp. 1-13, en. 2020. DOI: 10.1016/j.chemosphere.2020.127882.

[14] U. Javed, R. A. Khushnood, S. A. Memon, F. E. Jalal y M. S. Zafar, "Sustainable incorporation of lime-bentonite 
clay composite for production of ecofriendly bricks", $J$. Clean. Prod., vol. 263, 1 ag. 2020. DOI: 10.1016/j.jclepro.2020.121469.

[15] M. N. Rojas-Valencia, y E. Aquino-Bolaños, "Sustainable adobe bricks with construction wastes", Proceedings of Institution of Civil Engineers: Waste and Resource Management, vol. 169, no. 4, pp. 158-165, 1 nov. 2016. DOI: 10.1680/jwarm.16.00014.

[16] K. D. González-Velandia, R. Sánchez-Bernal, D. J. Pita-Castañeda y L. F. Pérez-Navarro, "Caracterización de las propiedades mecánicas de un ladrillo no estructural de tierra como soporte de material vegetal en muros verdes", Ingeniería Investigación y Tecnología, pp. 2-9, Octubre 2019. DOI: 10.22201/fi.25940732e.2019.20n3.030.

[17] R. Sánchez-Bernal, D. J. Pita-Castañeda, K. D. González-Velandia y J. A. Hormaza-Verdugo, "Análisis de mezclas de residuos sólidos orgánicos empleadas en la fabricación de ladrillos ecológicos no estructurales", $R C A$, vol. 53, no. 1, 2019. DOI: 10.15359/rca.53-1.2.

[18] B. S. Mohammed, M. Aswin y Vethamoorty, "Properties and Structural Behavior of Sawdust Interlocking Bricks". En: N. A. W.A. Zawawi (ed.), Engineering Challenges for Sustainable Future - Proceedings of the 3rd International Conference on Civil, Offshore and Environmental Engineering (ICCOEE) 2016, pp. 437-442, 2016. DOI: 10.1201/b21942-89.

[19] S. H. Adnan, M. A. b. Mohd Nar, M. H. Osman, W. A. Wan Jusoh, Z. Jamellodin, M. N. A. Wahee Anuar, "The study on used of tropical wood sawdust as a replacement fine aggregates in concrete mix", International Journal of Advanced Science and Technology, vol. 29, no. 6s, pp. 1542-1548, 2020. Disponible en: http://sersc.org/journals/index.php/IJAST/article/ view/9295/5138.

[20] M. F. Omar, M. A. H. Abdullah, N. A. Rashid y A. L. Abdul Rani, "Partially Replacement of Cement by Sawdust and Fly Ash in Lightweight Foam Concrete", IOP Conference Series: Materials Science and Engineering, vol. 743, no. 012035, pp. 1-7, 18 mar. 2020. DOI: 10.1088/1757-899X/743/1/012035.

[21] H. A. Colorado y G. I. Echeverri-Lopera, "The solid waste in Colombia analyzed via gross domestic product: Towards a sustainable economy", Revista Facultad de In- geniería Universidad de Antioquia, no. 96, pp. 51-63, feb. 2020. DOI: 10.17533/udea.redin.20191046.

[22] J. Kumar, S. Kumar y S. S. Basarkar, "Concrete using agro-waste as fine aggregate for sustainable built environment - A review", Int. J. Sustain. Built Environ., vol. 5, no. 2, pp. 312-333, 2016. DOI: 10.1016/j.ijsbe.2016.06.003.

[23] N. A. León, N. R. Rojas-Reyes, B. Umbarila-Suárez y R. O. Bustamente, "Evaluación experimental de unidades sílico-calcáreas a partir de escoria de alto horno y cal hidráulica para mampostería", DYNA, vol. 76, no. 160, pp. 247-254, 2009. Disponible en: https://revistas.unal. edu.co/index.php/dyna/article/view/13667/14462.

[24] H. Y. Liu, H. S. Wu y C. P. Chou, "Study on engineering and thermal properties of environment-friendly lightweight brick made from Kinmen oyster shells \& sorghum waste", Constr Build Mater., vol. 246, 20 jun. 2020. DOI: 10.1016 / j.conbuildmat.2020.118367.

[25] R. Ahmad, R. M. Nor y S. A. Ismail, "Use of sawdust as admixture in cement-sand brick", Pertanika J. Sci. Technol., vol. 25, no. supl. may., pp. 205-210, may. 2017.

[26] M. Čáchová, D. Koňáková, E. Vejmelková, M. Keppert y R. Černý, "Moisture properties of the lightweight brick body", AIP Conference Proceedings 1738, 280009, pp. 1-5, 8 jun. 2016. DOI: 10.1063/1.4952069.

[27] G. Cultrone, I. Aurrekoetxea, C. Casado y A. Arizzi, "Sawdust recycling in the production of lightweight bricks: How the amount of additive an the firing temperature influence the physical properties of the bricks", Constr Build Mater., vol. 235, 117436, pp. 1-13, 28 feb. 2020. DOI: 10.1016/j.conbuildmat.2019.117436.

[28] P. Muñoz-Velasco, M. Mendivil-Giro, M. P. Morales y L. Muñoz-Velasco, "Eco-fired clay bricks made by adding spent coffee grounds: a sustainable way to improve buildings insulation", Mater Struct, vol. 49, pp. 641650, en. 2016. DOI: 10.1617/s11527-015-0525-6.

[29] T. Dias-Silva, H. Martins de Paula, D. da Silva, I. Mazon-Carvalho, J. T. Fonte y R. Rodrigues-Pereira, "Uso de granulado de borracha em substituição parcial ao agregado miúdo na produção de tijolos ecológicos", $M a$ téria (Rio J.), vol. 2, no. 4, 2 oct. 2017. DOI: 10.1590/ s1517-707620170004.0239. 
[30] M. Lamrani, M. Mansour, N. Laaroussi y M. Khalfaoui, "Thermal study of clay bricks reinforced by three ecological materials in south of morocco", Energy Procedia, vol. 156, pp. 273-277, 2019. DOI: 10.1016/j.egypro.2018.11.141.

[31] H. Limami, I. Manssouri, K. Cherkaoui y A. Khaldoun, "Physicochemical, Mechanical and Thermal Performance of Lightweight Bricks with Recycled Date Pits Waste Additives”, J. Build. Eng., vol. 34, 2020. DOI: 10.1016/j.jobe.2020.101867.

[32] M. Martins, M. Ferreira Leão de Oliveira, R. C. da Conceição-Ribeiro, D. Cruz-Bastos y M. Gomes de Oliveira, "Ecological bricks from dimension stone waste and polyester resin”, Constr Build Mater., vol. 232, en. 2020. DOI: 10.1016/j.conbuildmat.2019.117252.

[33] O. T. Maza-Ignacio, V. G. Jiménez-Quero, J. Guerrero-Paz y P. Montes-García, "Recycling untreated sugarcane bagasse ash and industrial wastes for the preparation of resistant, lightweight and ecological fired bricks", Constr Build Mater., vol. 234, feb. 2020. DOI: 10.1016/j. conbuildmat.2019.117314.

[34] S. M. A. C. de Freitas, L. N. Sousa, P. Diniz, M. E. Martins y P. S. Assis, "Steel slag and iron ore tailings to produce solid brick", Clean Techn Environ Policy, vol. 20, pp. 1087-1095, 2018. DOI: 10.1007/s10098-018-1513-7.

[35] P. Indhiradevi, P. Manikandan, K. Rajkumar y S. Logeswaran, "A comparative study on usage of cowdung ash and wood ash as partial", Materials Today: Proceedings, 2020. DOI: 10.1016/j.matpr.2020.06.355.

[36] A. I. Hafez, M. M. A. Khedr, R. M. Osman, R. Sabry y M. S. Mohammed, "A comparative investigation of the unit cost for the preparation of modified sand and clay bricks from rice husk waste", J. Build. Eng., vol. 32, 2020. DOI: 10.1016/j.jobe.2020.101765.

[37] L. Liu, X. Cheng, X. Miao, Y. Shi, M. Zhang, M. Guo, F. Cheng y M. Zhang, "Preparation and characterization of majority solid waste based eco-unburned permeable bricks", Constr Build Mater., vol. 259, 2020. DOI: 10.1016/j.conbuildmat.2020.120400.

[38] P. Muñoz-Velasco, V. Letelier, M. A. Bustamante, J. Marcos-Ortega y J. G. Sepúlveda, "Assessment of mechanical, thermal, mineral and physical properties of fired clay brick made by mixing kaolinitic red clay and paper pulp residues", Appl. Clay Sci., vol. 198, pp. 1-10, 15 November 2020. DOI: 10.1016/j.clay.2020.105847.

[39] C. L. Susilawati, P. K. Yulius Suni y E. Tjandra, "Lockbrick system technology is an ecological building material innovation", IOP Conf. Ser.: Earth Environ. Sci., vol. 419, 2020. DOI: 10.1088/1755-1315 / 419/1/012005.

[40] R. M. Nidzam, I. Norsalisma y J. Mungai Kinuthia, "Strength and environmental evaluation of stabilised Clay-PFA eco-friendly bricks", Constr Build Mater., vol. 125, pp. 964-973, 2016. DOI: 10.1016/j.conbuildmat.2016.08.114.

[41] M. F. de Souza, J. Soriano y M. T. Ospina, "Resistência à compressão e viabilidade econômica de blocos de concreto dosado com resíduos de tijolos cerâmicos", $M a$ téria (Rio J.), vol. 23, no. 3, 2018. DOI: 10.1590/s1517707620180003.0537.

[42] J. Zhang, R. S. Srinivasan y C. Peng, "Ecological assessment of clay brick manufacturing in china using emergy analysis", Buildings, vol. 10, no. 11, pp. 1-17, 2020. DOI: 10.3390/buildings 10110190 .

[43] M. F. Serrano-Guzmán, D. D. Pérez-Ruiz, L. M. Torrado-Gómez y N. D. Hernández, "Residuos inertes para la preparación de ladrillos con material reciclable: una práctica”, Industrial Data, vol. 20, no. 1, pp. 131-138, 2017. DOI: 10.15381/idata.v20i1.13507.

[44] A. Barragán-Alturo, N. G. Duran-Siachoque, K. A. Figueroa-Gonzalez y M. X. Rovayo-Novoa, "Ecobloque estructural para vivienda de interés rural: Un aporte para las comunidades del Alto Magdalena-Colombia”, Lampsakos, no. 17, pp. 29-39, 2017. DOI: $10.21501 / 21454086.2214$.

[45] G. M. Viegas, C. Walsh y M. V. Barros, "Evaluación cualicuantitativa de aislaciones térmicas alternativas para viviendas. El caso de la agricultura familiar", Revista INVI, vol. 31, no. 86, pp. 89-117, 2016. DOI: 10.4067/ S0718-83582016000100004.

[46] A. B. Acevedo y F. N. Zora-Mejía, “Características estructurales de escuelas colombianas de pórticos de hormigón reforzado con mampostería no reforzada”, Ingeniería y Ciencia, vol. 13, no. 25, pp. 209-227, 2017. DOI: 10.17230/ingciencia.13.25.9. 
S. P. Muñoz-Pérez et al. Fabricación de ladrillos ecológicos en muros no estructurales... ARTÍCULO DE REVISIÓN

[47] J. Carrillo y G. González, "Influencia de la mampostería no reforzada en el comportamiento inelástico de pórticos de concreto", DYNA, vol. 74, no. 152, pp. 217-227, 2007. Disponible en: https://revistas.unal.edu.co/index. php/dyna/article/view/925/1359

[48] S. Pujol y M. E. Rodríguez, "Evaluación del comportamiento de muros no estructurales en edificios de la Ciudad de México en el terremoto del 19 de septiembre 2017”, Ingeniería sísmica, no. 101, pp. 53-66, 2020. DOI: 10.18867/ris.101.529.
[49] A. Aviram, J. W. Badillo, J. A. Prieto y J. D. Jaramillo, "Unreinforced brick masonry wall collapse investigation under moderate wind loads", Rev. Ing. Constr., vol. 34, no. 1, 2019. DOI: 10.4067/S0718-50732019000100065.

[50] S. Constantinescu, "Non-bearing masonry walls behavior and influence to high reinforced concrete buildings", Adv. Sci. Technol. Eng. Syst. J., vol. 3, no. 6, pp. 111-117, 2018. DOI: 10.25046/aj030612. 